\title{
A Word of Caution about Many Labs 4: If You Fail to Follow Your Preregistered Plan, You May Fail to Find a Real Effect
}

\author{
Armand Chatard \\ Université de Poitiers, CNRS, Poitiers, France
}

\author{
Gilad Hirschberger \\ Interdisciplinary Center (IDC) Herzliya, Israel
}

\author{
Tom Pyszczynski \\ University of Colorado, Springs, USA
}

\begin{abstract}
A team of 37 researchers (Many Labs 4; Klein et al., 2019) has recently reported that it has failed to replicate the effect of mortality salience on worldview defense - a classic finding from terror management theory (TMT). This collaborative project (21 labs, $N=2220$ ) has the potential to provide useful information regarding the robustness of an often-replicated and influential empirical finding. However, Klein et al. (2019) deviated from their preregistered plan by including smaller samples than specified in their pre-registration. This undisclosed deviation from their plan is problematic because it undermines the potential benefits of pre-registration; indeed, the negative results of their meta-analyses appear to be predominantly driven by small studies. We reanalyzed the Many Labs 4 data after excluding studies that did not meet the preregistered minimum sample size (40 participants per cell). Results showed that the data actually do replicate the original study. This successful replication emerged only in the expert advice variation of these studies, which addresses one of the purposes of this study by showing that replication is more likely to occur when researchers follow the advice of researchers with considerable experience in this domain. We discuss the importance of following preregistered plans to avoid misleading conclusions and potential issues involved with literal replications of effects that may depend on societal zeitgeist at the time data are collected.
\end{abstract}

Keywords: Terror Management Theory, replication, Many Labs 4, failure to replicate, small sample studies, reanalysis, preregistered

Note: This manuscript is a preprint. It is susceptible to change as it has not yet been peer-reviewed or published in a journal.

Acknowledgements: We thank colleagues working on terror management theory for taking part in a group discussion thread related to this project.

Author contributions: A. Chatard reanalyzed the Many Labs 4 data and drafted the report. G. Hirschberger and Tom

\section{Introduction}

Terror Management Theory (TMT; Greenberg, Pyszczynski, \& Solomon, 1986; Solomon, Greenberg, \& Pyszczynski, 1991), developed over 30 years ago, has been an influential framework in social psychology that has been applied to diverse aspects of human behavior. According to TMT, cultural worldviews (e.g., religious beliefs, political attitudes) serve an adaptive function: they help human beings manage the fear of death. Therefore, individuals should be especially motivated to defend and preserve their cultural worldviews after being reminded of death (the mortality salience hypothesis). Hundreds of published studies, in more than 36 countries,
Pyszczynski provided critical revisions and contributed to writing.

Corresponding Author: Armand Chatard, Université de Poitiers, Centre de Recherches sur la Cognition et l'Apprentissage CNRS 7295, Maison des Sciences de l'Homme et de la Société, Bat A5, 5 rue Théodore Lefebvre, TSA 21103, 86073 Poitiers, France. E-mail: armand.chatard@univ-poitiers.fr

have supported this mortality salience hypothesis (for an overview of 30 years of research on TMT, see Pyszczynski, Solomon, \& Greenberg, 2015; for a meta-analysis, see Burke, Martens, \& Faucher, 2010).

Based on the accumulated evidence, it was somewhat surprising to learn that a team of 37 researchers recently failed to replicate an often-replicated TMT effect (Greenberg, Pyszczynski, Solomon, Simon, \& Breus, 1994) in a large-scale effort (Many Labs 4; Klein et al., 2019). Indeed, Klein et al., (2019) reported that the Many Labs 4 (ML4) researchers could not replicate Greenberg et al.'s (1994) original effect even when they followed the protocol advised by TMT experts. This failure to 
replicate is noteworthy because the ML4 study was well powered (21 labs, $\mathrm{N}=2220)$, providing the capacity to yield precise estimates of effect sizes. Moreover, the ML4 study was preregistered, which reduces the likelihood of publication bias and flexibility in data analysis (p-hacking). We believe that multiple labs replication projects have the potential to make valuable contributions to the literature if properly conducted and carefully interpreted.

Prior to publication or peer review, a preliminary report of the ML4 research was posted on a public repository (https://psyarxiv.com/vef2c) and it has already generated a great deal of discussion and controversy on various blogs and social media sites. Some twitter users quickly proclaimed the "death of terror management theory." Within a week of its online appearance, an article appeared on the Psychology Today website claiming that the ML4 findings invalidate the argument that a lack of expertise and experience is sometimes responsible for failures to replicate previously published findings (Danvers, 2019); more recently a discussion of this project appeared on an Apple Podcast (https://blackgoat.podbean.com/e/the-expertiseof-death/) that drew similarly strong conclusions from this paper. We have also received a great deal of informal correspondence about this work. We would normally not comment on unpublished research that has not been peer reviewed, because we think it inappropriate to broadly disseminate research prior to scientific vetting. However, because of the attention the ML4 paper has already received, and of course because it deals directly with our own work, we deemed it important to point to some serious problems with the way this research was conducted and analyzed, and to document the very different conclusions that result when the data is properly analyzed.

Before discussing these problems, we want to be clear that we would be among the first to acknowledge that there is much that is not yet understood about terror management processes in general and mortality salience effects in particular. Over the past 33 years our studies have often yielded surprising and sometimes inconsistent findings. We have viewed these inconsistencies as challenges to specify moderators that determine when reminders of death do and do not produce particular effects. Sometimes those moderators followed directly from TMT, as in the case of individual differences in self-esteem, worldview threat, and other variables central to the theory. Sometimes the data led us to discover that things like delay and distraction or an experiential mindset are essential ingredients for particular forms of defensive responses to death, which led to theoretical refinements, in this case, the proximal-distal model of responses to conscious and non-conscious threats (Pyszczynski, Greenberg, \& Solomon, 1999). Indeed the history of TMT research could be viewed as a gradual process of discovering moderators and mediators of these effects. Nonetheless, there is much that is not yet fully understood, and we enthusiastically endorse research that points to situations in which previously obtained results do and do not replicate.

The purpose of the ML4 project was to determine if getting advice and input from those with considerable experience and expertise within a research domain increases the likelihood of successful replication. Thus, the ML4 team contacted some of us and asked for our advice on how to best conduct a mortality salience experiment. Though they followed most of the advice we provided, they did not heed our concerns about possible problems with the dependent variable, responses to essays praising or criticizing the United States in the months after Donald Trump was elected president. We explained that the intense anger and dismay that many (but certainly not all) Americans felt about Trump's election, which was likely especially prominent among the liberal samples typically found on university campuses, made us uncertain about how those essays would impinge on participants' cultural worldviews. Defense against threats to one's cultural worldview is the conceptual variable that TMT posits must be threatened for defensive responses to occur, and we were uncertain whether these essays would do this in the wake of Trump's election and in an era of intense emotional political division. Though the ML4 team acknowledged this concern, they used this measure anyway, probably because they wanted to conduct a literal replication of an operationalization of the effect of MS on a widely used way of assessing worldview defense. This would be a reasonable thing to do if one's interest is restricted to questions of literal replication, but responses to the posting of this study suggest that many observers do not take such subtleties into account. In cases like this, in which a dependent measure is likely to be dependent on the cultural zeitgeist of the time, it important to carefully consider whether a particular operationalization is likely to tap into the same psychological processes that it did in the past. Still, given how widely used this 
measure has been in previous TMT research, assessing whether a literal replication of this effect would occur nowadays is of interest. But findings need to be interpreted in light of consideration of the different cultural zeitgeists.

These concerns notwithstanding, another possible explanation for the failure to replicate Greenberg et al.'s (1994) finding is that the original effect was a false positive; that is, reminders of death do not affect evaluations of those who praise or criticize one's culture. This implies that the many previous studies demonstrating this effect reflect unstable effect estimates or inappropriate statistical practices. TMT studies, conducted before the so-called "replication crisis," were underpowered compared to current standards. The norm and common research practice in social psychology when most of these studies were done was to conduct a series of small-scale conceptual replication studies and to publish only studies that showed significant effects, leading to exaggerated effect sizes (publication bias). As TMT researchers, we are well placed to know that our studies were not an exception; indeed, editors frequently advised authors to drop studies from papers that were not fully consistent with hypotheses or try new analyses that would "remove the warts" from data. That being said, we were quite confident that the original effect published more than 25 years ago (Greenberg et al., 1994), that was replicated numerous times (e.g., Arndt et al., 1997; Burke et al., 2010; Deschesne et al., 3003; Greenberg et al., 1994) was real.

We were, therefore, initially puzzled by the results of ML4 suggesting that it might have been no more than a false positive. So we carefully examined the ML4 paper, the preregistered data plan, and the data set itself; we suspect we looked into this much more carefully than the vast majority of the readers of their online posting, especially those who have drawn broad conclusions about the empirical viability of TMT from this paper. In doing so, we were quick to discover some rather startling irregularities in the way the data were analyzed.

Because the ML4 project was an ambitious effort that included 21 labs and over 2200 participants, and the ML4 team appears to have made a genuine effort to follow most of the suggestions we gleaned from our years of experience studying these processes, we focus on the data they collected (with the caveats noted above). Because pre-registration is central to the standards for psychological research championed by the Open Science Movement and Many Labs Project, we carefully followed their preregistered protocol in our reexamination of the data from this study.

\section{Data Analytic Problems}

Careful inspection of the preregistered protocol, the R-script, and the data analyses reported in their paper reveal that Klein et al. (2019) deviated from their pre-registered protocol and failed to mention these deviations in their paper. Some deviations were relatively minor, but others were not. Of greatest significance, many studies included in the ML4 meta-analysis included a smaller than specified sample size (i.e., less than 40 participants per cell). In the Sample size section of the preregistered plan (https://osf.io/4xx6w), the ML4 researchers wrote: "The goal is to collect at least 40 participants per cell of that experiment. Therefore, labs (...) will be expected to collect data from at least 80 participants total." In the same way, in the Sample size rational section of the preregistered plan, the authors wrote: "40 participants per cell (80 participants per lab) is a guideline used in prior Many Labs projects (Klein et al., 2014; Ebersole et al., 2016) that is a compromise between minimally informative power within each site, while allowing many universities/labs to participate in the project. That said, 80 is viewed as a minimum (...)." These plans are echoed in the draft of the paper they posted online. Therefore, it is very clear from the preregistration plan that each study included in their analyses should include at least 80 participants per site, which the ML4 team stated is required to provide "minimally informative power" to replicate the original effect within each site. Because the researchers anticipated excluding a number of participants in some preregistered analyses, i.e., all participants who were not White Americans (Exclusion set 2) and those who did not strongly identify with the US (Exclusion set 3 ), the inclusion criterion of 80 participants per lab is arguably a bare minimum to have enough power to replicate the original effect. Surprisingly, Klein et al. (2019) deviated from their preregistration plan and included 8 studies (38\% of the total number of studies) in their meta-analysis that did not meet the " 80 participants per lab" inclusion criterion. Three studies used in their analysis included less than 20 participants per cell, providing very imprecise estimates of effect sizes (Simmons, Nelson, \& Simonsohn, 2011).

The deviation from the preregistered plan is problematic, not only because it was not transparently disclosed in the research report (https://psyarxiv.com/vef2c), but because small 
studies are often more heterogeneous and less precise than large ones (IntHout, Ioannidis, Borm, \& Goeman, 2015). Indeed, this is one of the problems that the Many Labs Project was designed to solve. Thus, even in the absence of publication bias, the inclusion of a large proportion of small studies in a meta-analysis can seriously impact the precision of the metaanalysis results (Lin, 2018; Nüesch et al., 2010). This may lead researchers to misleadingly conclude that there is an effect when there is not, or that there is no effect when in fact there is one. An examination of the ML4 data confirmed this fear. The overall effect size was greater for the 13 studies that met the inclusion criteria (Hedges' $g=0.10, S E=0.06$ ) than for the 8 studies that did not (Hedges' $g=-0.06$, $S E=0.10)$. A Mann-Whitney test showed that this difference was significant, $U=24.0, p=$ .046 , Rank-Biserial Correlation $=.54,95 \mathrm{CI}[.08$, $.80]$. This raises the possibility that the alleged failure to replicate was due to the inclusion of underpowered, heterogeneous and imprecise small studies in the meta-analysis. This prompted us to independently reanalyze the data of the ML4 project.

Our reanalysis of the ML4 project followed the guidelines of academic journals proposing a registered report format, such as Psychological Science and Journal of Experimental Social Psychology, as well as the recipe for conducting a convincing replication suggested by Brandt et al., (2014). To follow the criteria the authors provided in their preregistration, only the 13 studies that met the inclusion criterion of "at least 80 participants per lab" were retained for the confirmatory analyses. As in the ML4 study, the overall effect size was also computed separately for the labs that followed their own protocol (in house labs, $k=7$ ) and for those that followed the protocol advised by TMT experts (expert labs, $k=6$ ). Following the preregistration plan, replication success was defined as a significant effect $(p<$ $.05)$ in the expected direction, with an effect size greater than 0.10 (expressed in Cohen's $d$ units).

\section{Method}

The method is described in the Many Labs 4 project (https://psyarxiv.com/vef2c). A total of 2220 participants, participating in studies conducted by 21 labs, were randomly assigned to a mortality salience or a TV control condition. The main dependent variable was the preference for pro- versus anti- American essay. Among the 13 labs that met the inclusion criterion of at least 80 participants $(\mathrm{N}=1782)$, there were 7 In House labs and 6 Expert advised labs.

As explained in the ML4 paper, the Expert labs followed the procedural advice provided by TMT experts. Most importantly, a delay was included between the mortality salience manipulation and the worldview defense measure. To do this, participants were asked to fill-in two unrelated questionnaires to create a delay. Although this may seem a mundane detail, previous research and theorizing on TMT indicates that a delay is necessary to obtain the original effect (Arndt 1997; Pyszczynski et al., 1999; Burke et al., 2010). Within the in house-designed condition, the labs constructed their own procedure based solely on the paper that described the original effect to be replicated (Greenberg at al., 1994). There was much more heterogeneity in procedures among the In House labs, compared to the Expert advised labs. For example, all of the In House labs except for one (six out of seven) used no delay between the mortality salience manipulation and the worldview defense measure, and the other used a considerably shorter delay than that used in the expert versions.

Following the ML4 team's preregistered plan, we repeated our confirmatory analyses under three different exclusion criteria suggested by TMT experts: Exclusion Set 1: Include all participants who completed the priming procedure and all items used for computing the dependent variable $(\mathrm{N}=$ 1782 for the 13 labs meeting the preregistered inclusion criterion). Exclusion Set 2: All prior exclusions, and further exclude participants who did not identify as White Americans $(\mathrm{N}=1513$ for the 13 labs meeting the preregistered inclusion criterion). Exclusion Set 3: All prior exclusions, and further exclude participants who responded lower than 7 out of 9 on the American Identity item ("How important to you is your identity as an American?") ( $\mathrm{N}=1372$ for the 13 labs meeting the preregistered inclusion criterion). Because the data required to make these exclusions were often not collected at In House replication sites we followed Klein et al. (2019) in using only Exclusion Set 1 for analyses of In House data. Thus, our analyses mirror the inclusion criteria used by Klein et al., for both expert and in house studies. Indeed, our re-analysis followed the statistical procedures in all ways except that we followed their preregistered criteria for sample sizes.

The ML4 researchers did not specify whether they would use one-tailed or two-tailed tests in their preregistration plan. However, a 
one-tailed $t$ test is justified because the preregistered hypothesis was unidirectional. As stated in the preregistration plan (https://osf.io/4xx6w): "the general guideline for the present project will consider a study successfully replicated if it yields a significant effect $(p<.05)$ in the expected direction". Clearly, finding less pro-US bias in the mortality salience condition would not be a successful replication of the original finding. Moreover, one-tailed tests are more powerful than two-tailed tests (Lakens, 2016), which may compensate for reduced power in our reanalysis due to the exclusion of small studies. For these reasons, we report hereafter one-sided tests and $90 \%$ confidence intervals.

The data and code used in this reanalysis are posted at the project's OSF repository (https://osf.io/6v4kf/).

\section{Results}

As in the ML4 project, we conducted a random-effects meta-analysis to examine whether we could replicate the classic mortality salience effect on worldview defense. However, this time, the data were analyzed as planned in the preregistration form. That is, we selected only the 13 studies that met the preregistered minimum sample size $(\mathrm{N}>80$ participants per study) for the meta-analysis. We used the Maximum Likelihood method because it is the method by default in the MetaSEM $\mathrm{R}$ package (Cheung, 2014), used by Klein et al. (2019). The random-effects meta-analysis produces the grand mean effect size across all sites and versions. Table 1 presents the overall effect size for the different exclusion criteria considered in the preregistration plan. For comparison purpose, Table 1 also presents the overall effect size for the meta-analysis presented by Klein et al. (2019) that included the small $\mathrm{n}$ studies.

As shown in Table 1, results indicated that when the meta-analysis was conducted in accordance with the preregistered protocol $(\mathrm{k}=$ 13), the classic TMT effect was successfully replicated, at least when some exclusion criteria were considered (Exclusion Set 2). When only White Americans were selected in the analysis (Exclusion Set 2), the mortality salience effect was significant in the expected direction $(p<$ .05 ), with an effect size greater than 0.10 (see Figure 1). When only White Americans strongly identified to the US were selected in the analysis (Exclusion Set 3), the mortality salience effect was marginally significant $(p<$ .09 ), with an effect size greater than 0.10 .

These findings contradict the main conclusion of the ML4 study (Klein et al.,
2019). When the data were analyzed as planned in the preregistration form, the results of the ML4 study were more consistent with TMT than initially thought. Though one might question the exclusion criteria, these were preregistered and suggested by the expert researchers because they follow from previous theory and research on these issues, and assessing the impact of their advice was the major goal of the ML4 study. But one of the major goals for this research, as stated by the authors, was to determine if expert advice affects the likelihood of successful replication.

Accordingly, we examined whether the overall effect size was larger in the Expert labs condition than in the In House labs condition. Table 2 indicates that, whatever the exclusion set considered, the overall effect size was larger when the replicators followed the procedure advised by TMT experts. For Exclusion Set 2 and 3 , the effects found in the Expert labs condition reached the bar set for a successful replication according to the preregistration plan (see Figure 2). In contrast, the overall effect size in the In House labs condition did not qualify as a successful replication according to the preregistration plan. In sum, results of confirmatory analyses showed that the data actually provided support for the original effect (Greenberg et al., 1994), but this occurred only when the replicators followed the protocol advised by TMT experts.

The ML4 preregistered data plan had an additional discrepancy from what was reported in the ML4 paper. Although both the ML4 paper and the preregistered data plan specify a sample size of 40 participants per cell, the preregistered plan includes a data exclusion section that states, "Samples will be included as long as they collect at least 60 participants by the time data collection ends." We therefore conducted supplementary analyses with this more liberal cutoff $(\mathrm{N}>60)$ and obtained similar findings. The overall effect size for mortality salience effect was not significant in the in house lab condition (Hedges' $g=0.03$, $90 \% \mathrm{CI}=[-0.08,0.14], S E=0.06, Z=0.45, p=$ 0.32 ). The overall effect size for mortality salience effect was not significant in the expert lab condition for Exclusion Set 1 (Hedges' $g=$ $0.08,90 \% \mathrm{CI}=[-0.04,0.21], S E=0.07, Z=$ $1.11, p=0.14)$. However, the overall effect size for mortality salience effect was significant in the expert lab condition for Exclusion Sets 2 and 3 (Hedges' $g=0.21,90 \% \mathrm{CI}=[0.04,0.37]$, $S E=0.10, Z=2.06, p=0.02$, and Hedges' $g=$ $0.23,90 \%$ CI $=[0.02,0.44], S E=0.13, Z=$ 1.77, $p=0.04$, respectively). Again, this 
suggests that the negative results of Klein et al.'s (2019) meta-analysis were likely driven by a few small, heterogeneous, and imprecise studies that should not have been included in the meta-analysis if the authors had conducted the studies as planned.

\section{Discussion}

The present reanalysis provides new insights regarding why the ML4 study seemed to have failed to replicate a classic terror management effect. It turns out that the authors of the Many Labs 4 study did not follow their own preregistered plan. They included several studies with a smaller sample size than expected in their meta-analysis. This deviation was not clearly disclosed in the research report that was made public (Klein et al., 2019). The authors reported the analyses on all the 21 studies, even those that did not meet the preregistered inclusion criterion, as if they were confirmatory analyses. However, these analyses deviated from the preregistered plan and should have been reported, if anything, as exploratory analyses. Here we show that when the ML4 data are analyzed as planed, then the effects are quite different from those reported by Klein et al. (2019). Indeed, the data of this massive replication effort provide substantial support for the original effect (Greenberg et al., 1994); importantly, this effect emerged only when the replicators followed the procedures advocated by TMT experts. The findings of our reanalysis have important implications for both preregistered replications (meta-science) and terror management theory.

\section{Implications for meta-science}

The main goal of replication attempts, such as Many Labs, is to reveal type I error (false positives). Our reanalysis of the ML4 study suggests that it is also important to closely follow pre-registered plans because of the chance of producing a type II error or false negative results - concluding that there is no effect when there actually is one. Previous research suggests that many false positives in psychology may be due to a failure to differentiate confirmatory from exploratory analyses, or, in other words, prediction from postdiction (Nosek, Ebersole, DeHaven, \& Mellor, 2018). Our reanalysis complements and extends this reasoning by showing that a failure to differentiate confirmatory from exploratory analyses may also lead researchers to mistakenly conclude that there is no effect when there actually is one. A false negative is problematic in science because it could lead scientists to abandon a prolific and potentially important field of inquiry on the basis of erroneous conclusions.

A central lesson from our reanalysis of Many Labs 4 concerns the problem of smallstudy effects in meta-analyses. The generic term "small-study effects" is often used to indicate that studies with smaller sample sizes often show different, usually larger, treatment effects than studies with larger sample sizes. The most widely recognized reason is publication bias (Schwarzer, Carpenter, \& Rücker, 2015). However, because the ML4 study was preregistered, publication bias cannot account for the small-study effects found in the present data. Another (often underestimated) reason for small-study-effects is that small studies often show very heterogeneous and imprecise data points in meta-analysis (IntHout et al., 2015). A set of imprecise data points is unlikely to provide an accurate estimate of an effect size in meta-analysis (Lin, 2018; Nüesch et al., 2010). Our reanalysis showed that the inclusion of a large proportion of small studies in the ML4 meta-analysis created a real statistical issue. The effect size of the studies with less than 40 participants per cell was significantly lower than the effect size of the studies that met the preregistered minimum sample size. Thus, although the ML4 replication study has an overall large $\mathrm{N}$, small samples from each site distorted the data, leading the researchers to misleadingly conclude that they failed to replicate the original effect. An important contribution of the present reanalysis is to show that the influence of small studies on estimated effect sizes in meta-analysis should be routinely assessed even in preregistered studies to avoid either false negative or false positive conclusions.

In the context of the current replication crisis, publishing failed replications may be especially appealing, because it is consistent with the provocative current zeitgeist that most studies conducted before the replication crisis are unreliable. It has recently been argued that flexibility in data analysis makes it easy to publish false negative replication results, while maintaining the appearance of methodological rigor (Bryan, Yeager, \& O’Brien, 2019). We wholeheartedly agree with the basic premises of Many Labs that past research should be carefully scrutinized and replicated (e.g., Tello, Harika-Germaneau, Serra, Jaafari, \& Chatard, 2020). Yet, our reanalysis of ML4 stands as a clear signal to be careful not to throw out the baby with the bathwater. Overzealous attempts to dispose of existing bodies of knowledge in 
social psychology may inadvertently and ironically encourage questionable research practices in replication attempts, at a time when it is especially important that such attempts uphold the highest and most stringent standards.

The failure of ML4 to adhere to the high standards that they advocate indicates that replication attempts should be critically examined like any other research. Although we do not believe that Klein et al. (2019) engaged in deceptive practices (reverse p-hacking, obfuscating results) to produce non-significant results, we do believe that even 37 established scientists can make significant errors. In particular, the failure of in-house labs to follow the procedures explicitly described in many terror management studies and meta-analyses (in particular the delay following the MS condition), and the failure of ML4 to follow their own preregistered protocol has led to the erroneous conclusion that an effect does not exist when their own data shows that it does. Such mistakes are preventable if the procedures and preregistered analyses are transparently reported and followed to the letter. One of the most important contributions of our reanalysis is in underscoring the importance of full transparency in reporting the results of preregistered studies, and in demonstrating how deviations, especially unreported deviations, from the preregistration plan may skew the results and lead to erroneous conclusions.

Although the ostensible death of terror management has spread like wildfire on psychology social media sites, leading some to conclude that terror management does not work and that there is no "secret sauce" (i.e., expert advantage) in producing terror management effects (Danvers, 2019), our reanalysis of the ML4 study indicates that such conclusions are unwarranted. Our results indicate that the original effect was replicated, but only when the replicators followed the procedure advocated by TMT experts but not when researchers followed their own procedure. The failure of in-house labs is disconcerting but not completely surprising given all that has been learned about the processes involved in mortality salience effects and the procedures needed to find them since the publication of the Greenberg et al. (1994) study 25 years ago. This finding regarding the importance of expert advice has important implications for replication science. It demonstrates for the first time the critical importance of involving original authors, or experts, in the replication process.

\section{Implications for terror management theory}

Our results also have implications for terror management theory. A first implication concerns the robustness of the original effect. Some of the original authors expressed doubts that the original effect on this particular dependent measure published over 25 years ago could still be replicated nowadays, because the original effect was obtained in a more harmonious political climate and the replication was conducted in the year after the election of President D. Trump (cf. an email exchange between R. Klein and J. Greenberg). This change in political climate could have potentially changed what it means to be "proUS", the dependent measure used in Greenberg et al. (1994) and in ML4. Our reanalysis of the Many Labs 4 study suggests that these concerns did not bear out. The original effect was clearly replicated in this high-powered preregistered study, at least among White American participants and when advice based on years of experience conducting mortality salience studies was followed. This indicates that the original effect is, indeed, quite robust. The fact that the original effect was consistently found among White American participants, but not among the full sample including minority group members, suggests that the election of President Trump could have played some role in affecting the responses of minority group members. Further studies are needed to examine this possibility.

A second implication for terror management theory concerns the importance of the delay between the mortality salience induction and essay evaluation. The proximaldistal addition to TMT (Pyszczynski et al., 1999) posits that effects of mortality salience on worldview defense are more pronounced when time has passed or participants have been distracted from the issue of death because different defenses are used when thoughts of death are on the fringes of consciousness than when they are in focal attention. To create a delay, TMT researchers routinely ask participants to complete filler tasks. A metaanalysis of terror management effects shows not only that delay is important for obtaining the effect, but that long delays produced stronger effects than short delays (Burke et al., 2010). In the ML4 study, all labs that followed the procedure advised by TMT experts included two filler tasks. In contrast, the in-house labs deviated from this procedure and included either a shorter delay ( 7 labs) or no delay at all (5 labs). Our reanalysis indicates that the original effect was replicated among the labs with sufficient sample sizes that followed the 
procedure advised by TMT experts, but not among those that created their own procedure. Since one of the main differences between the two protocols was the delay, this finding provides indirect evidence for the notion that worldview defense is a distal terror management defense that is activated after a delay, when thoughts of death are not in current focal attention (Pyszczynski et al., 1999).

A final implication of our reanalysis of the Many Labs 4 study concerns the precision of the effect size estimate of mortality salience. Recent research suggests that, compared to preregistered multiple lab replication studies, meta-analyses of published findings overestimate true effect sizes because of pervasive publication bias (Kvarven, Strømland, \& Johannesson, 2019). Kvarven et al. (2019) compared meta-analyses and multiple lab replication studies of 17 effects in psychology. They found that effect sizes differed significantly and systematically: those reported in meta-analyses were about three times larger than those found in multiple lab replication studies. In our reanalysis of the ML4 study, we found a mean effect size (expressed in Hedges's $g$ units) across the 6 expert labs that varied between 0.10 and 0.27 depending on the exclusion set considered. This effect size estimate is similar to the one found by Kvarven et al. (2019) for other multiple lab replication studies in psychology, but substantially lower than the one reported in a previous metaanalysis of terror management effects (Burke et al., 2010). Thus, our reanalysis of the ML4 study may help better estimate the true effect size of terror management studies.

\section{Limitations}

The most significant limitation of the present reanalysis of the ML4 study is that it is based on a limited set of studies $(k=13)$, which precluded us from conducting moderation analysis. Thus, even if our findings clearly suggests that the mortality salience effect was replicated among the labs that followed the procedure advised by terror management experts, future studies are needed to further examine the moderation effect by lab expertise. Although tangential to terror management theory, this is an important question for future multiple replication studies.

\section{Conclusion}

The 37 researchers from the Many Labs 4 study should be commended for conducting a large-scale preregistered replication study on an important effect in social psychology. However, the failure to follow their (highly justified) preregistered protocol has led to incorrect conclusions that have become widely publicized and have inadvertently done more damage than good to our common goal of improving psychological science. Many Labs should be held to the same standards as any other research endeavor in social psychology and it is crucial that in future replication attempts the preregistered protocol is stringently followed to reduce researcher degrees of freedom and insure that both type I and type II errors are reduced to a minimum.

\section{References}

Arndt, J., Greenberg, J., Pyszczynski, T., \& Solomon, S. (1997). Subliminal exposure to death-related stimuli increases defense of the cultural worldview. Psychological Science, 8, 379-385.

Brandt, M. J., IJzerman, H., Dijksterhuis, A., Farach, F. J., Geller, J., Giner-Sorolla, R., ... \& Van't Veer, A. (2014). The replication recipe: What makes for a convincing replication? Journal of Experimental Social Psychology, 50, 217-224.

Bryan, C., Yeager, D. S., \& O’Brien, J. (2019). Replicator degrees of freedom allow publication of misleading failures to replicate. Proceedings of the National Academy of Sciences, 116, 25535-25545.

Burke, B. L., Martens, A., \& Faucher, E. H. (2010). Two decades of terror management theory: A metaanalysis of mortality salience research. Personality and Social Psychology Review, 14, 155195.

Cheung, M. W.-L. (2014b). metaSEM: Meta-Analysis using Structural Equation Modeling, Version 0.9. Singapore: R package.

Danvers, A. (2019, December 13). Rejecting the "secret sauce excuse". [Blog post]. Retrieved from https://www.psychologytoday.com/us/blog/how-do-you-know/201912/rejecting-the-secretsauce-excuse 
Dechesne, M., Pyszczynski, T., Arndt, J., Ransom, S., Sheldon, K. M., Van Knippenberg, A., \& Janssen, J. (2003). Literal and symbolic immortality: The effect of evidence of literal immortality on self-esteem striving in response to mortality salience. Journal of personality and social psychology, 84, 722-737.

Ebersole, C. R., Atherton, O. E., Belanger, A. L., Skulborstad, H. M., Allen, J. M., Banks, J. B., ... \& Brown, E. R. (2016). Many Labs 3: Evaluating participant pool quality across the academic semester via replication. Journal of Experimental Social Psychology, 67, 68-82.

Greenberg, J., Pyszczynski, T., Solomon, S., Simon, L., \& Breus, M. (1994). Role of consciousness and accessibility of death-related thoughts in mortality salience effects. Journal of Personality and Social Psychology, 67, 627-637.

Greenberg, J., Pyszczynski, T., \& Solomon, S. (1986). The causes and consequences of a need for selfesteem: A terror management theory. In Public self and private self (pp. 189-212). Springer, New York, NY.

IntHout, J., Ioannidis, J. P., Borm, G. F., \& Goeman, J. J. (2015). Small studies are more heterogeneous than large ones: a meta-meta-analysis. Journal of clinical epidemiology, 68, 860-869.

Klein, R. A., Ratliff, K. A., Vianello, M., Adams Jr, R. B., Bahník, Š., Bernstein, M. J., ... \& Nosek, B. (2014). Investigating variation in replicability. Social Psychology, 45, pp. 142-152.

Klein, R. A., Cook, C. L., Ebersole, C. R., Vitiello, C. A., Nosek, B. A., Chartier, C. R., ... Ratliff, K. A. (2019, December 11). Many Labs 4: Failure to replicate mortality salience effect with and without original author involvement. https://doi.org/10.31234/osf.io/vef2c

Kvarven, A., Strømland, E., \& Johannesson, M. (2019). Comparing meta-analyses and preregistered multiple-laboratory replication projects. Nature Human Behaviour, 1-12.

Lakens, D. (2016). One-sided tests: Efficient and underused [Blog post]. Retrieved from http://daniellakens.blogspot.com/2016/03/one-sided-tests-efficient-and-underused.html

Lin, L. (2018). Bias caused by sampling error in meta-analysis with small sample sizes. Plos One 13(9): e0204056.

Nosek, B. A., Ebersole, C. R., DeHaven, A. C., \& Mellor, D. T. (2018). The preregistration revolution. Proceedings of the National Academy of Sciences, 115, 2600-2606.

Nüesch, E., Trelle, S., Reichenbach, S., Rutjes, A. W., Tschannen, B., Altman, D. G., ... \& Jüni, P. (2010). Small study effects in meta-analyses of osteoarthritis trials: meta-epidemiological study. BMJ, 341, c3515.

Pyszczynski, T., Greenberg, J., \& Solomon, S. (1999). A dual-process model of defense against conscious and unconscious death-related thoughts: an extension of terror management theory. Psychological Review, 106, 835-845.

Pyszczynski, T., Solomon, S., \& Greenberg, J. (2015). Thirty years of terror management theory: From genesis to revelation. Advances in Experimental Social Psychology, 52, 1-70.

Simmons, J. P., Nelson, L. D., \& Simonsohn, U. (2011). False-positive psychology: Undisclosed flexibility in data collection and analysis allows presenting anything as significant. Psychological Science, 22, 1359-1366.

Schwarzer, G., Carpenter, J. R., \& Rücker, G. (2015). Small-study effects in meta-analysis. In Metaanalysis with $R$ (pp. 107-141). Springer, Cham.

Solomon, S., Greenberg, J., \& Pyszczynski, T. (1991). A terror management theory of social behavior: The psychological functions of self-esteem and cultural worldviews. Advances in Experimental Social Psychology, 24, 93-159.

Tello, N., Harika-Germaneau, G., Serra, W., Jaafari, N., \& Chatard, A. (2020). Forecasting a fatal decision: Direct replication of the predictive validity of the suicide-implicit association test. Psychological Science, 31, 65-74. 
Table 1. Random-Effects models (with $\mathrm{k}=13$ studies conducted as preregistered and with $\mathrm{k}=21$ studies as reported by Klein et al., 2019)

Effect size, $90 \% \mathrm{CI}$ and $p$-value

Exclusion Set 1 (used for both in-house and expert labs)

$k=13$ studies

Hedges' $g=0.06,90 \% \mathrm{CI}=[-0.03,0.15], S E=0.05, Z=1.17, p=0.13$

$k=21$ studies

Hedges' $g=0.03,90 \% \mathrm{CI}=[-0.05,0.10], S E=0.05, Z=0.58, p=0.29$

Exclusion Set 2 (used for expert labs only)

$k=13$ studies Hedges' $g=0.13,90 \% \mathrm{CI}=[0.01,0.26], S E=0.08, Z=1.72, p=0.045$

$k=21$ studies $\quad H_{e d g e s}^{\prime} g=0.06,90 \% \mathrm{CI}=[-0.04,0.15], S E=0.06, Z=1.03, p=0.16$

Exclusion Set 3 (used for expert labs only)

$k=13$ samples $\quad$ Hedges $^{\prime} g=0.10,90 \% \mathrm{CI}=[-0.02,0.22], S E=0.07, Z=1.40, p=0.09$

$k=21$ studies $\quad H e d g e s^{\prime} g=0.04,90 \% \mathrm{CI}=[-0.05,0.14], S E=0.06, Z=0.73, p=0.24$

Note. Tau² Estimator: Maximum-Likelihood. p-values are unidirectional (one-tailed). 
Figure 1. Forest plot of observed effects sizes (Exclusion Set 2, $\mathrm{k}=13$ studies with $\mathrm{N}>80$, as preregistered)

\begin{tabular}{|c|c|c|}
\hline University of Florida & $\mapsto$ & $-0.17[-0.38,0.04]$ \\
\hline Pace University & $\longmapsto$ & $-0.08[-0.48,0.33]$ \\
\hline Ithaca College & $\mapsto$ & $-0.05[-0.30,0.20]$ \\
\hline Worcester Polytechnic Institute & $\longmapsto$ & $-0.01[-0.29,0.27]$ \\
\hline Pacific Lutheran University & $\mapsto$ & $-0.01[-0.21,0.20]$ \\
\hline Wesleyan University & $\mapsto$ & $0.06[-0.19,0.31]$ \\
\hline Occidental College & $\longrightarrow$ & $0.09[-0.41,0.59]$ \\
\hline Virginia Commonwealth University & $\longrightarrow$ & $0.15[-0.42,0.72]$ \\
\hline University of Pennsylvania & $\mapsto$ & $0.18[-0.18,0.53]$ \\
\hline The College of New Jersey & $\stackrel{-1}{\longrightarrow}$ & $0.21[-0.12,0.54]$ \\
\hline Brigham Young University & $\longrightarrow$ & $0.37[-0.02,0.76]$ \\
\hline University of Illinois & $\longmapsto$ & $0.74[0.37,1.10]$ \\
\hline University of California, Riverside & $\longmapsto$ & $1.69[0.94,2.44]$ \\
\hline \multirow[t]{3}{*}{ RE Model } & 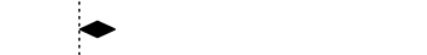 & $0.13[0.01,0.26]$ \\
\hline & $T$ & $\neg$ \\
\hline & 2 & 3 \\
\hline
\end{tabular}


Table 2. Random-Effects model $(\mathrm{k}=13$ studies with $\mathrm{N}>80$, as preregistered)

Effect size, $90 \% \mathrm{CI}$ and $p$-value

Exclusion Set 1 (used for both in-house and expert labs)

In house $(\mathrm{k}=7) \quad$ Hedges $^{\prime} g=0.06,90 \% \mathrm{CI}=[-0.08,0.19], S E=0.08, Z=0.69, p=0.25$

Expert $(\mathrm{k}=6) \quad$ Hedges $^{\prime} \mathrm{g}=0.10,90 \% \mathrm{CI}=[-0.04,0.23], S E=0.08, Z=1.21, p=0.12$

Exclusion Set 2 (used for expert labs only)

Expert $(\mathrm{k}=6) \quad$ Hedges' $g=0.27,90 \% \mathrm{CI}=[0.06,0.48], S E=0.13, Z=2.08, p=0.02$

Exclusion Set 3 (used for expert labs only)

Expert $(\mathrm{k}=6) \quad$ Hedges $^{\prime} g=0.25,90 \% \mathrm{CI}=[0.02,0.48], S E=0.14, Z=1.76, p=0.04$

Note. Tau² Estimator: Maximum-Likelihood. p-values are unidirectional (one-tailed). 
Figure 2. Forest plot of observed effects sizes in the Expert labs condition for Exclusion Set 2 (top panel) and Exclusion Set 3 (bottom panel) ( $\mathrm{k}=6$ studies, with $\mathrm{N}>80$, as preregistered)

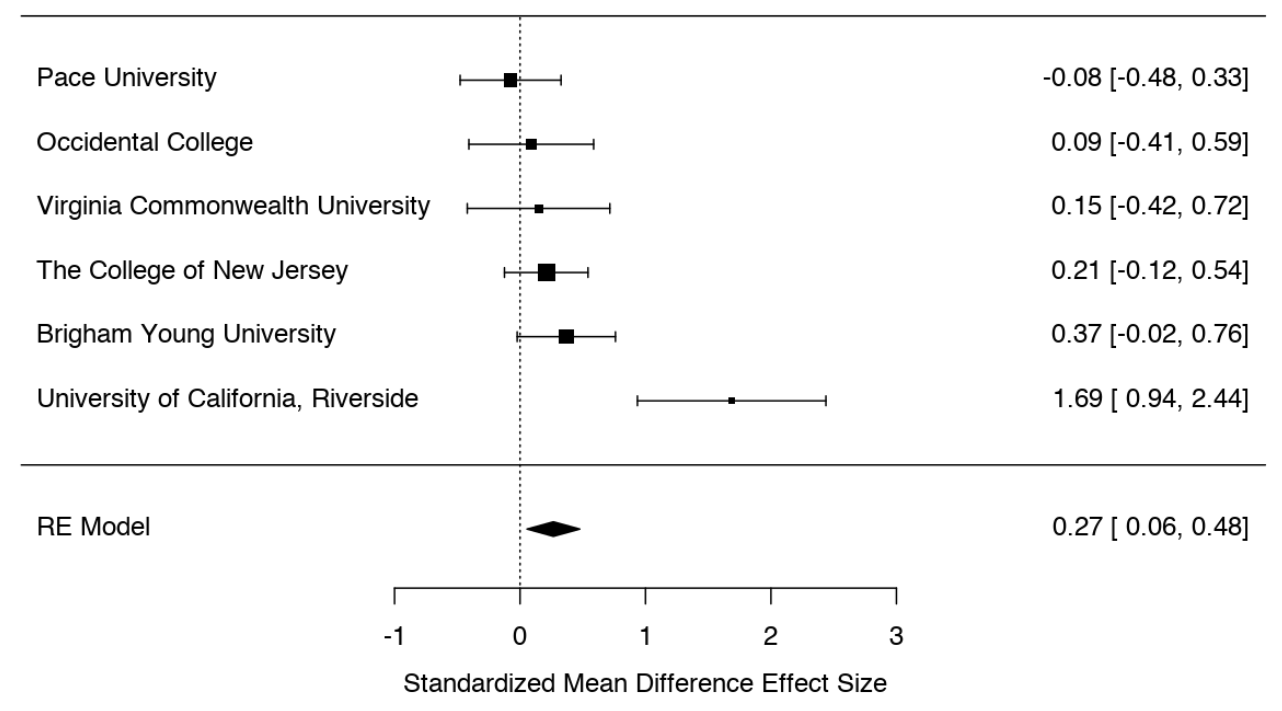

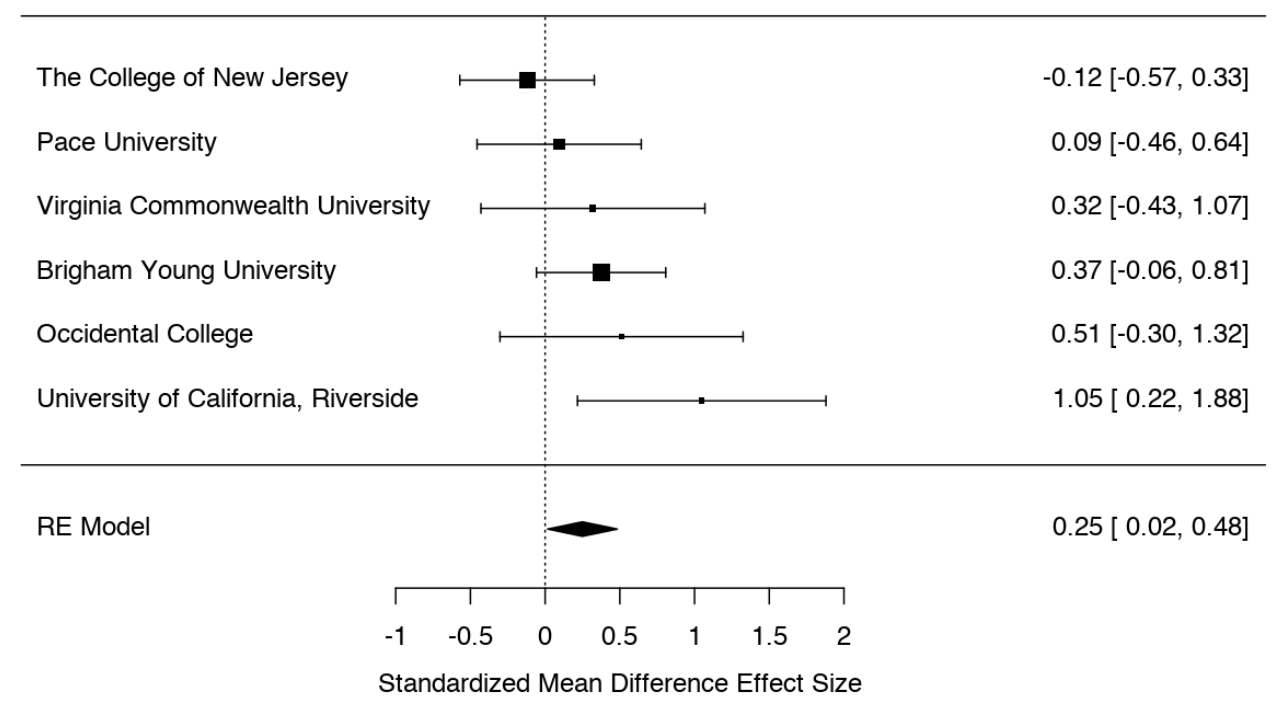

